\title{
Social Adaptive Navigation Support for Open Corpus Electronic Textbooks
}

\author{
Peter Brusilovsky ${ }^{1,2}$, Girish Chavan ${ }^{1}$, and Rosta Farzan ${ }^{2}$ \\ ${ }^{1}$ School of Information Sciences and ${ }^{2}$ Intelligent Systems Program \\ University of Pittsburgh, Pittsburgh PA 15260, USA \\ peterb@pitt.edu, gchavan@mail.sis.pitt.edu, rosta@cs.pitt.edu
}

\begin{abstract}
Closed corpus AH systems demonstrate what is possible to achieve with adaptive hypermedia technologies; however they are impractical for dealing with the large volume of open corpus resources. Our system, Knowledge Sea II, presented in this paper explores social adaptive navigation support, an approach for providing personalized guidance in the open corpus context. Following the ideas of social navigation, we have attempted to organize a personalized navigation support that is based on past learners' interaction with the system. The social adaptive navigation support implemented in our system was considered quite useful by students participating in the classroom study of Knowledge Sea II. At the same time, some user comments indicated the need to provide more powerful navigation support.
\end{abstract}

\section{Introduction}

The electronic textbook is an emerging form for delivering structured educational resources over the Web [10]. Web-based electronic textbooks successfully attempt to resemble classic textbooks, a proven form for expressing and communicating knowledge. Web-based electronic textbooks are attractive for both providers (publishers, teachers) and consumers (students). Teachers use online textbooks to deliver structured content to their students with minimal efforts. Publishers explore the use of Web-based textbooks to deliver the content faster while decreasing the costs for maintaining the rapidly changing information. Students embrace the online form since it can be accessed "anytime, anywhere". They also appreciate that the Web can bring more information to their fingertips than traditional paper-based sources can. It's quite typical nowadays for one to find a selection of electronic textbooks on the Web, in a number of popular subjects - ranging from a simple, hierarchicallystructured set of HTML pages prepared by individual teachers to professionallyproduced electronic copies of popular, printed textbooks.

Wider choice is beneficial - potentially it gives more opportunities for everyone to locate their most-personally-relevant learning objects. Unfortunately the benefits of online access come with its price - an information overload. As we have found in the course of our earlier research [7], it is very hard for regular students to select relevant reading fragments on a specific topic from multiple online textbooks. It is known that 
users with weak knowledge of the subject (that is usually the case for students) have navigation problems in educational hypermedia even when dealing with a single hyperdocument [3]. The problems can be resolved to some extent by navigation support that is aimed to help students in locating the most relevant fragments of educational content. In a traditional educational context this navigation support is provided by a teacher who assigns relevant readings for every lecture or topic of the course being taught. An even higher level of navigation support can be provided by adaptive educational hypermedia systems [2]. These systems rely on individual student models and expert knowledge about educational material to guide the students to the most relevant fragments.

The problem addressed in this paper is that traditional, expert-based approaches can only provide guidance within a relatively limited set of resources (so-called Closed Corpus) and can't cope with the increasing volume of online information (known as Open Corpus). No teacher can recommend the most relevant reading from dozens of relevant online sources. Adaptive educational hypermedia systems can potentially deal with large volumes of information but detailed content knowledge is required. While in some application areas such as aircraft maintenance [5] it is feasible to have a team of experts to encode the complete content knowledge about thousands and thousands of available content fragments, the educational economies of most fields preclude this investment of time and money.

Our system, Knowledge Sea II presented in this paper, explores an alternative approach for providing personalized guidance to students. Following the idea of social navigation [15] we have attempted to organize a personalized navigation support that is based on past learners' interaction with the system. We call this social adaptive navigation support (SANS). Unlike traditional adaptive navigation support that relies on expert-provided knowledge about each resource, social adaptive navigation support relies on the collective knowledge of a large community of learners gathered through different forms of feedback. This paper discusses the idea of SANS and presents the Knowledge Sea II system that implements some simple form of SANS. In addition, we report the results of our classroom study of the system and discuss these results in the context of our future work.

\section{Navigating Multiple Educational Resources in Closed Corpus and Open Corpus Hypermedia}

As we have pointed out in the introduction, adaptive hypermedia systems can provide a high level of navigation support by helping users of educational hypermedia find educational material that is most relevant in the context of their current knowledge and goals. While early adaptive hypermedia systems developed for the E-Learning context $[9 ; 12]$ have focused on providing adaptive navigation support in a single hyperdocument, more recent systems have demonstrated an ability to deal with multiple independent sources. Such systems as InterBook [6], KBS-Hyperbook [17], and SIGUE [11] have explored several ways to integrate the content of several electronic textbooks or Web sites. 
For example, InterBook suggested the metaphor of a bookshelf - a collection of independently developed electronic textbooks on the same subject. To allow students to navigate seamlessly between different textbooks, InterBook introduced conceptbased navigation though the glossary [8]. Each concept used for indexing bookshelf content in InterBook can be visualized as a glossary page. The glossary page for a specific concept shows some authored definition of the concept and automatically generates links to all pages in all textbooks on that shelf that are related to this concept. Glossary pages support goal-based access to educational material by providing the student with one-click access to all resources on that shelf that either present each specific concept or require knowledge about the concept. It also provides an additional level of knowledge-based navigation support by indicating pages that present no new information to the student, pages that are ready to be learned, and pages beyond the student's current learning ability.

Unfortunately, the InterBook's adaptive navigation support power is available only within a relatively small set of documents that were structured and indexed with concepts at design time. Modern AH systems are predominantly closed corpus adaptive hypermedia since the document space of these adaptive systems is a closed set of information items manually indexed by domain experts. KBS-Hyperbook [18], and SIGUE [11] are two of only a few systems that have attempted to deal with an open corpus such as the Web. However, even in KBS-Hyperbook and SIGUE the ability to handle open corpus resources comes for a price: each Web page added to these systems has to be manually indexed with domain model concepts.

To support students in navigating open corpus educational resources, we have to use methods that do not require manual indexing of content. One of the fields that can provide relevant methods is information retrieval. Information retrieval approaches are mostly based on automatic content indexing on the level of keywords. Information retrieval methods are far from reaching the precision of classic adaptive hypermedia in determining the most relevant pages, yet they are able to operate with huge amounts of "open Web" content [1].

In our earlier Knowledge Sea project [7] we explored the possibility of automatically structuring a reasonable amount of the open corpus Web resources. Knowledge Sea provides structured access to several online textbooks on $\mathrm{C}$ programming language in the context of an introductory programming course. Knowledge Sea used a keyword-level automatic page analysis and self-organized maps (SOM) to allocate pages of several hierarchically-structured Web textbooks (open corpus) and lecture handouts (closed corpus) on an 8 by 8 grid. We have relied on the remarkable ability of SOM to group similar pages in the same cells as well as to place reasonably similar groups of pages in neighboring cells. SOM allowed us to provide what we called map-based navigation for multiple educational resources. It resembles InterBook's concept-based navigation to some extent, but does not require manual indexing or structuring. Cell keywords (identified by SOM) and lecture landmarks were placed on the map to help students finding cells that are close to their current goal. Each cell can be "opened" in a separate window that will show the content of the cell - a list of links to pages from multiple tutorials. This allowed the students to navigate "horizontally" between tutorials in addition to hierarchical navigation supported by each tutorial. 
The system was called Knowledge Sea because the cells on the map were colored using several shades of blue to express the amount of resources under the cell. Similar to real Ocean maps, deeper color saturation indicated that the Information Sea under the cells was "deeper" - i.e., more resources were available. The system was evaluated in several classroom studies. The students praised highly the ability of Knowledge Sea to group similar resources together and to help them find relevant pages. Least appreciated feature of the system was the interface. A number of students have been confused by our attempt to use color saturation to indicate the depth of the Information Sea. In addition, a number of students have noted that Knowledge Sea provided no help in locating relevant resources within a cell. Since pages in a cell are grouped on the basis of keyword level similarity, very good pages can be located next to difficult-to-comprehend pages or pages that contain no useful information.

The goal of our more recent Knowledge Sea II system was to extend the power of Knowledge Sea by providing navigation support within a cell. Knowledge Sea II uses adaptive annotation technology that had already been used in InterBook in a very similar context. Our challenge was to replace the InterBook adaptation engine (based on closed corpus indexing) with an engine that could work with open corpus resources. In Knowledge Sea II we explored the possibility of building an adaptation engine based on the concepts of social navigation theory.

\section{Social Adaptive Navigation Support for Open Corpus Hypermedia}

Social navigation [15] is a new stream of research that explores methods for organizing users' explicit and implicit feedback, in order to support information navigation in a meaningful way. The field of social navigation began with a few now classic projects $[14 ; 21]$ that attempted to support a known social phenomenon: people tend to follow the "footprints" of other people. Another important feature of all social navigation systems is self-organization. Social navigation systems are able to work with little or no involvement of human administrators or experts. They are powered by a community of users. Properly organized community-powered systems such as Web auctions (www.ebay.com) or Weblogs (www.lifejournal.com) are known to be among the most successful Web applications. Self-organization is critical to the goals of our project. In an online educational context, self-organization means the ability to guide students to the most useful resources without the need for continuous involvement of human content experts to index resources. The feasibility of social navigation in the context of E-Learning has been explored in a few pioneer projects $[16 ; 19 ; 20]$. These projects provided an inspiration for our work. We have attempted however, to move one step further by developing a social adaptive navigation support technology that can navigate within much larger volumes of educational content.

The first version of Knowledge Sea II combines the concepts of group user modeling with the original "footprint" approach [21]. It uses the simplest implicit feedback: group traffic. For each tutorial page it counts how many times the users of the same group accessed it. Summing up group traffic for individual pages belonging to the same map cell, the system can also calculate group traffic for each cell. To keep 
the original ocean map metaphor, we have decided to visualize the cell traffic using multiple shades of blue. The more saturated the color is the more times the pages belonging to the cell were accessed. As a group of users navigates though pages of multiple electronic textbooks, the knowledge map becomes gradually darker. As we had expected, during the classroom study of Knowledge Sea II, we observed that cells with the most useful content become darker much faster. At the end of the course (Figure 1), from the teacher's point of view, the colors of the cells have remarkably well captured the usefulness of the map cells in the context of the given course. The darkest color indicated "treasure" cells with a good number of pages presenting important course topics. The cells that focus on topics not covered by the course have remained nearly white.

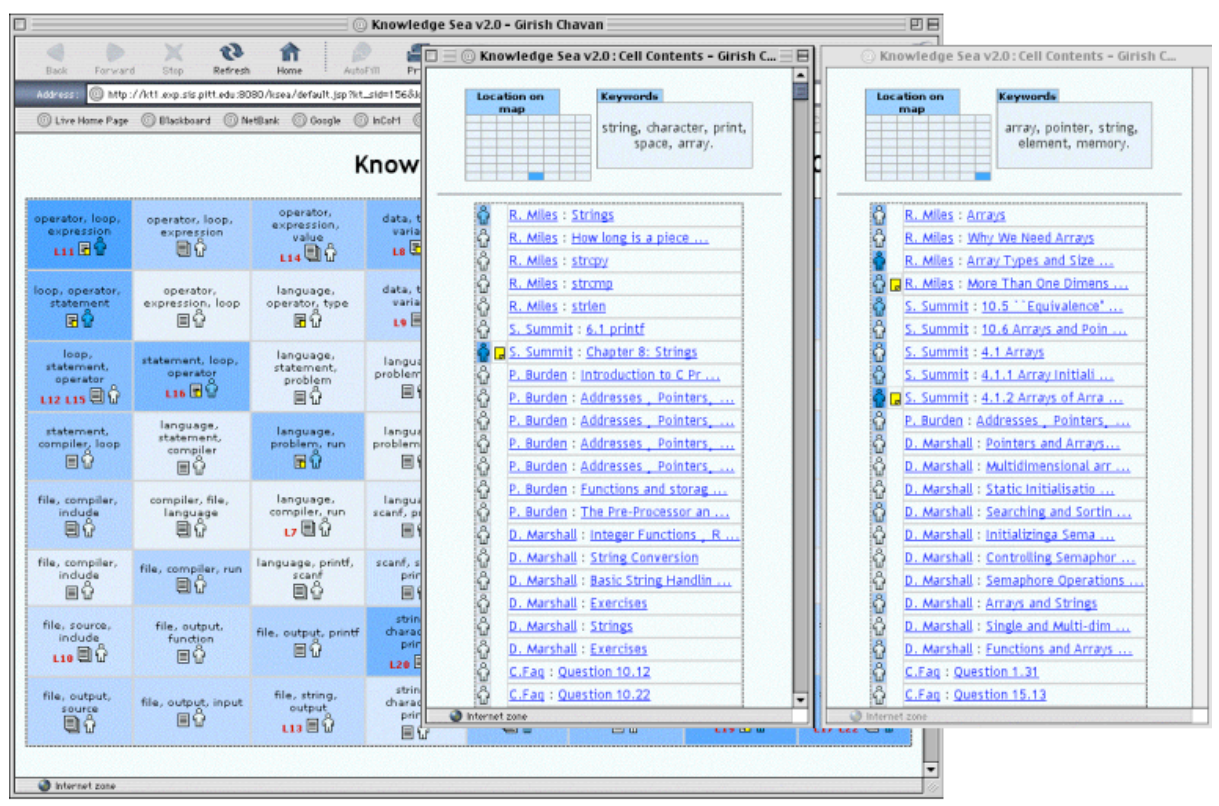

Fig. 1. The map view and two cell views in Knowledge Sea II

In addition to expressing the amount of group traffic through color saturation, each cell on the map shows four other cues. The list of top three cell keywords (identified by SOM) helps the students in finding cells by content. Lecture numbers play the role of landmarks allowing users to locate open corpus documents that are similar to wellknown closed corpus documents. The icon shaped as a stack of papers indicates the relative number of documents in the cell and serves as an anchor for accessing the cell. A small yellow note on this icon hints about the presence of user notes on some cell pages. The human-shaped icon indicates the number of user's own visits to the documents of the cells using the same metaphor - darker shades for more visits. This technique provides an extended form of history-based navigation support explored in some early educational adaptive hypermedia [13]. The user can see which pages are 
well explored and which are not. In addition, the colors of the background and the icon are generated to ensure their matching. As a result, if the user has visited the cell more than the average user of her group, the icon is darker than the background. If she has visited it less than average, the icon is lighter than the background. It allows the users to instantly distinguish potentially useful cells that they have underexplored as well as possibly over-explored cells.

The same traffic-based approach is used to annotate links within the map cell (Figure 1) and individual tutorial pages (Figure 2). Each link is annotated with a human-shaped blue icon on a blue background (right on Figure 1). The color of the icon shows user's own navigation history. The color of the background shows the cumulative navigation history of the whole class. In addition, a note-shaped icon indicates the presence of user notes on a page. The annotations in the cell window are generated by Knowledge Sea II. The annotations on tutorial pages are added by the AnnotatED service which processes all tutorial pages, adding annotations to visited and commented pages.

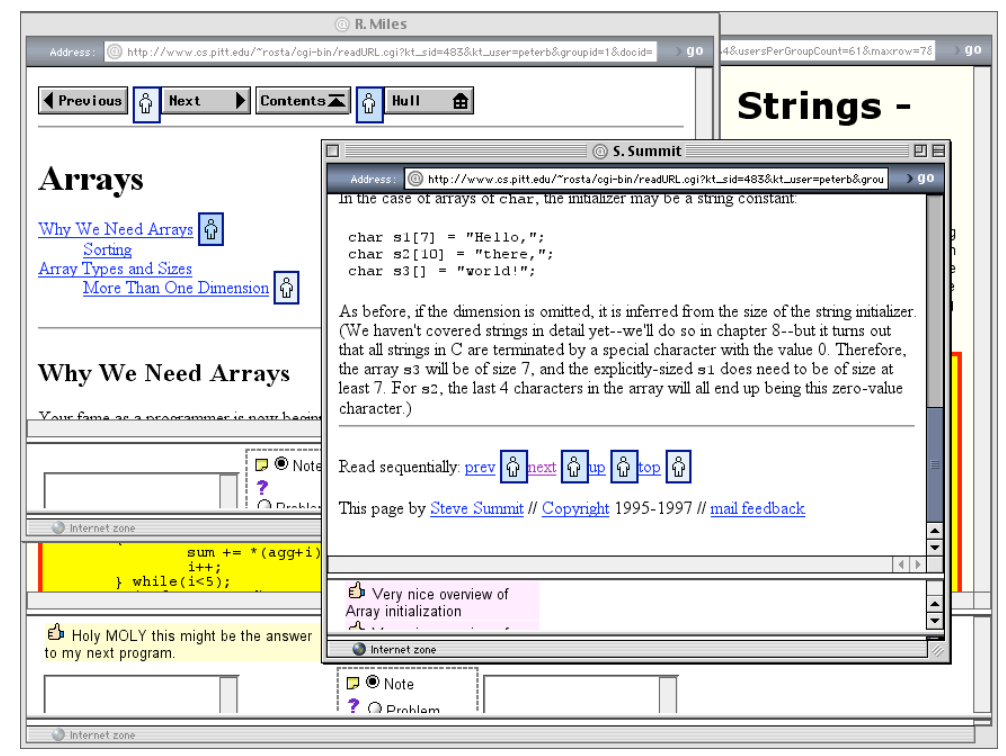

Fig. 2. Adaptive annotations indicating user and group traffic are added to tutorial pages by AnnotatED service.

\section{A Classroom Study of Social Adaptive Navigation Support}

We performed the first classroom study of Knowledge Sea II in a regular introductory programming course at the University of Pittsburgh. To compare two versions of the system we used the original Knowledge Sea system for the first 1/3 of the course and 
switched to Knowledge Sea II for the rest of the course. The teacher has briefly demonstrated the system during several lectures and invited students to use it in addition to the printed textbook used in class. Access to the Knowledge Sea map was provided through our KnowledgeTree course portal and tracked by the user model server [4]. In addition, links to several tutorial pages that the teacher considered useful were provided directly as an additional reading for some lectures. Out of 28 students in class 16 used the system. These students have made between 2 and 201 page hits. The average number of hits was 54 .

At the end of the semester, we asked the students who tried the system to fill out a non-mandatory questionnaire. The students were offered extra credit for participation. The questionnaire included 22 questions aimed to collect user feedback about various aspects of the system. Out of 16 eligible students 13 filled in the questionnaire. Due to lack of space, we present here only the results that are directly related to the topic of this paper - history-based social navigation support. Two groups of questions are relevant here. One group of four questions asked students to evaluate separately the idea of indicating individual and group traffic on the map and on individual pages with different shades of blue for icon/background annotations. We used a 4-point asymmetric scale where the answers ranged from highly positive (very clever interface feature) to negative (completely wrong idea). As we can see (Figure 3), the users regarded these features quite highly - about $80 \%$ of the users considered these features as either good or very good and none have considered it a bad idea. Splitting the users in two groups - those who accessed less than 30 pages ( 7 users) and those who accessed more ( 6 users), we have noted that the users who used the system more were much more positive about the color-based annotation. We were unable to find a significant difference due to the small number of subjects.

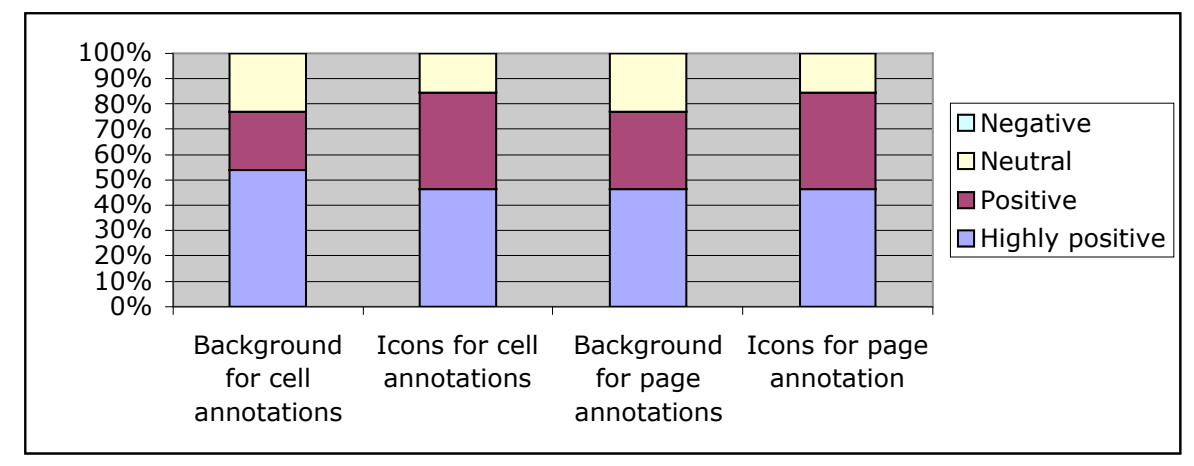

Fig. 3. Student's attitude to visualizing traffic with shades of blue, in four contexts

Another group of two questions was designed to assess what kind of traffic-based annotations the students prefer to have in general. For each of two contexts (map navigation and page navigation) we asked the users what kind of traffic they would like to see visualized in both map and page context. The answer options were: Only group traffic, Only user traffic, Both, or None. The answer analysis (Figure 4) allows 
making two interesting observations. First, students in general consider visualization of group traffic more useful than visualization of user traffic. While most users want to see either group traffic or both, merely one or two want to see only user traffic. Second, users who have reasonable experience working with the system (more than 30 hits) appreciate traffic visualization more than less experienced users. Moreover, most experienced users prefer to see both kinds of traffic visualized in both contexts.

Despite a very positive attitude toward traffic visualization in general and a specific preference for using icons and backgrounds colored in various shades of blue, some students indicated in their free form comment field that the traffic-based SANS is not sufficient for making informed navigation decisions. Two students noted that group traffic is not a reliable way to indicate page relevance. They wanted to see "good pages" that were considered useful for other group members. One student wanted to see what course lecture is relevant to each page listed.

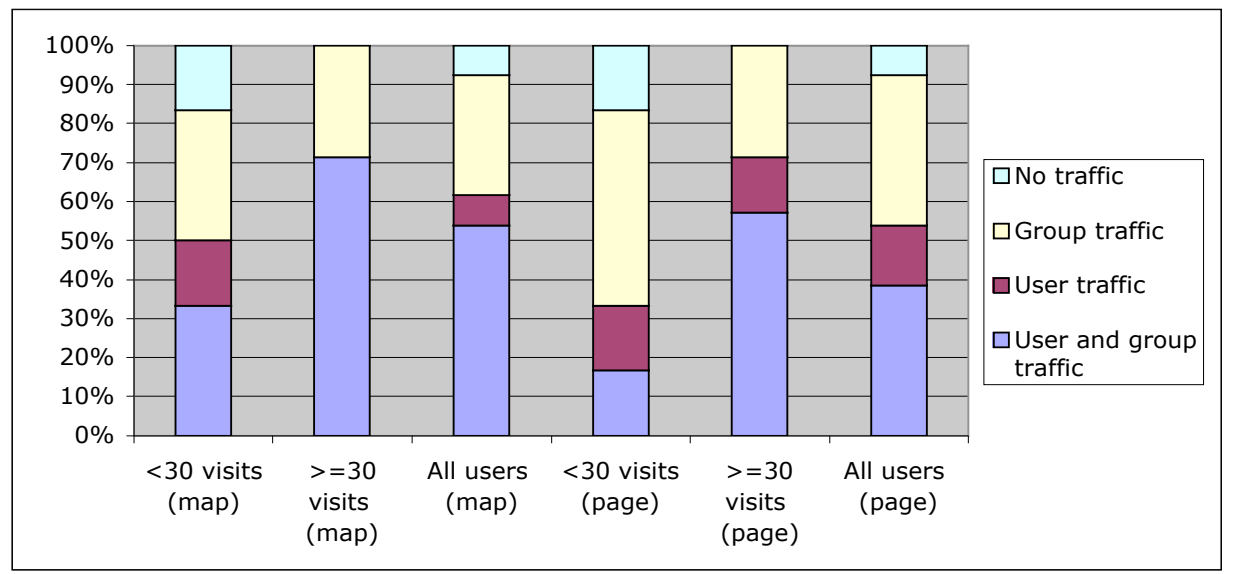

Fig. 4. User preferences about traffic visualization, split by user experience

In addition, we have compared the student attitude to the overall system's interface in the original Knowledge Sea system (as evaluated in several earlier studies) and the newer Knowledge Sea II. We were pleased to see that the student attitude toward the interface in the new system was quite a bit more positive than it was in our Knowledge Sea studies. Without any prompting, several students noted in their free form comment field that the new system is better and easier to use than the original Knowledge Sea. Since traffic-based adaptive annotations were the only significant change in the new system, we think that SANS has contributed to the increase of the user satisfaction with the new system.

\section{Conclusions and Future Work}

In this paper we have discussed problems encountered when providing adaptive navigation support for open corpus electronic textbooks. We have presented the 
Knowledge Sea II system that provides social adaptive navigation support - an open corpus adaptive navigation support based on the ideas of social navigation. While traffic-based SANS implemented in our system is relatively simple, it was considered quite useful by students participating in the classroom study of the system. At the same time, some user comments indicated the need to provide more powerful navigational support such as a method for the user to rate useful pages. We address these and other problems in the new version of Knowledge Sea II that is being evaluated in class this semester.

The main new idea that we are exploring in the new version is harvesting the feedback that the users provide through notes and comments that can be added to any open corpus page using AnnotatED service. In the version of the system described in this paper, notes were untyped and private (i.e., each student was able to see his or her notes only). In the newest version of the system we introduced typed notes and provided an option to make a note public. We hope that the analysis of students' extended commenting in the new version will give us some ideas on using the collective wisdom encapsulated in the comments for more precise SANS.

\section{References}

[1] Brin, S. and Page, L.: The anatomy of a large-scale hypertextual (Web) search engine. Computer Networks and ISDN Systems. 30, 1-7 (1998) 107-117

[2] Brusilovsky, P.: Adaptive hypermedia. User Modeling and User Adapted Interaction 11, 1/2 (2001) 87-110, available online at http://www.wkap.nl/oasis.htm/270983

[3] Brusilovsky, P.: Adaptive navigation support in educational hypermedia: the role of student knowledge level and the case for meta-adaptation. British Journal of Educational Technology 34, 4 (2003) 487-497

[4] Brusilovsky, P.: KnowledgeTree: A Distributed Architecture for Adaptive E-Learning. In: Proc. of The Thirteenth International World Wide Web Conference, WWW 2004 (Alternate track papers and posters), New York, NY, ACM Press (2004) 104-113

[5] Brusilovsky, P. and Cooper, D. W.: Domain, Task, and User Models for an Adaptive Hypermedia Performance Support System. In: Gil, Y. and Leake, D. B. (eds.) Proc. of 2002 International Conference on Intelligent User Interfaces, San Francisco, CA, (2002) 23-30, available online at http://www2.sis.pitt.edu/ peterb/papers/IUI02.pdf

[6] Brusilovsky, P., Eklund, J., and Schwarz, E.: Web-based education for all: A tool for developing adaptive courseware. Computer Networks and ISDN Systems. 30, 1-7 (1998) 291-300

[7] Brusilovsky, P. and Rizzo, R.: Using maps and landmarks for navigation between closed and open corpus hyperspace in Web-based education. The New Review of Hypermedia and Multimedia 9 (2002) 59-82

[8] Brusilovsky, P. and Schwarz, E.: Concept-based navigation in educational hypermedia and its implementation on WWW. In: Müldner, T. and Reeves, T. C. (eds.) Proc. of EDMEDIA/ED-TELECOM'97 - World Conference on Educational Multimedia/Hypermedia and World Conference on Educational Telecommunications, Calgary, Canada, AACE (1997) 112-117 
[9] Brusilovsky, P., Schwarz, E., and Weber, G.: ELM-ART: An intelligent tutoring system on World Wide Web. In: Frasson, C., Gauthier, G. and Lesgold, A. (eds.) Intelligent Tutoring Systems. Lecture Notes in Computer Science, Vol. 1086. Springer Verlag, Berlin (1996) 261-269

[10] Brusilovsky, P., Schwarz, E., and Weber, G.: Electronic textbooks on WWW: from static hypertext to interactivity and adaptivity. In: Khan, B. H. (ed.) Web Based Instruction. Educational Technology Publications, Englewood Cliffs, New Jersey (1997) 255-261

[11] Carmona, C., Bueno, D., Guzman, E., and Conejo, R.: SIGUE: Making Web Courses Adaptive. In: De Bra, P., Brusilovsky, P. and Conejo, R. (eds.) Proc. of Second International Conference on Adaptive Hypermedia and Adaptive Web-Based Systems (AH'2002) Proceedings, Málaga, Spain (2002) 376-379

[12] De Bra, P. M. E.: Teaching Hypertext and Hypermedia through the Web. Journal of Universal Computer Science 2, 12 (1996) 797-804, available online at http://www.iicm.edu/jucs_2_12/teaching_hypertext_and_hypermedia

[13] de La Passardiere, B. and Dufresne, A.: Adaptive navigational tools for educational hypermedia. In: Tomek, I. (ed.) Computer Assisted Learning. Springer-Verlag, Berlin (1992) 555-567

[14] Dieberger, A.: Supporting social navigation on the World Wide Web. International Journal of Human-Computer Interaction 46 (1997) 805-825

[15] Dieberger, A., Dourish, P., Höök, K., Resnick, P., and Wexelblat, A.: Social Navigation: Techniques for Building More Usable Systems. interactions 7, 6 (2000) 3645

[16] Dron, J., Boyne, C., and Mitchell, R.: Footpaths in the Stuff Swamp. In: Fowler, W. and Hasebrook, J. (eds.) Proc. of WebNet'2001, World Conference of the WWW and Internet, Orlando, FL, AACE (2001) 323-328

[17] Henze, N. and Nejdl, W.: Extendible adaptive hypermedia courseware: Integrating different courses and Web material. In: Brusilovsky, P., Stock, O. and Strapparava, C. (eds.) Adaptive Hypermedia and Adaptive Web-Based Systems. Lecture Notes in Computer Science, Springer-Verlag, Berlin (2000) 109-120

[18] Henze, N. and Nejdl, W.: Adaptation in open corpus hypermedia. International Journal of Artificial Intelligence in Education 12, 4 (2001) 325-350, available online at http://cbl.leeds.ac.uk/ijaied/abstracts/Vol_12/henze.html

[19] Kurhila, J., Miettinen, M., Nokelainen, P., and Tirri, H.: EDUCO - A collaborative learning environment based on social navigation. In: De Bra, P., Brusilovsky, P. and Conejo, R. (eds.) Proc. of Second International Conference on Adaptive Hypermedia and Adaptive Web-Based Systems (AH'2002), Málaga, Spain (2002) 242-252

[20] Mitsuhara, H., Ochi, Y., and Yano, Y.: Open-Ended Adaptive System for Facilitating Knowledge Construction in Web-Based Exploratory Learning. In: De Bra, P., Brusilovsky, P. and Conejo, R. (eds.) Proc. of Second International Conference on Adaptive Hypermedia and Adaptive Web-Based Systems (AH'2002), Málaga, Spain (2002) $547-550$

[21] Wexelblat, A. and Mayes, P.: Footprints: History-rich tools for information foraging. In: Proc. of ACM Conference on Human-Computer Interaction (CHI'99), Pittsburgh, PA (1999) 270-277 